\title{
STATUS OF FLAT ELECTRON BEAM PRODUCTION
}

\author{
D. Edwards, H. Edwards, N. Holtkamp, S. Nagaitsev, J. Santucci, FNAL* \\ R. Brinkmann, K. Desler, K. Flöttmann, DESY Hamburg \\ I. Bohnet, DESY Zeuthen, M. Ferrario, INFN-Frascati
}

\begin{abstract}
Last year at LINAC2000 [1] we reported our initial verification of the round beam (comparable transverse emittances) to flat beam (high transverse emittance ratio) transformation described by Brinkmann, Derbenev, and Flöttmann [2]. Further analysis of our data has confirmed that a transverse emittance ratio of approximately 50 was observed. Graphics representing observational detail are included here, and future plans outlined.
\end{abstract}

\section{INTRODUCTION}

Three years ago, Ya. Derbenev invented an optics maneuver for transforming a beam with a high ratio of horizontal to vertical emittance - a "flat beam" — to one with equal emittances in the transverse degrees-of-freedom-a "round beam".[3] High energy electron cooling at the TeV energy scale was the motivation.

Two years ago, R. Brinkmann and K. Flöttmann of DESY joined with Derbenev in a paper that reverses the process - obtain a flat beam from a round beam produced from the cathode of an electron gun.[2] Their idea was the simplification or even elimination of the electron damping ring in a linear collider project.

The intent of the present experiment was to verify the round-to-flat transformation and that was accomplished as reported earlier; further details and illustrations will be presented here.

\section{PRINCIPLE}

If the cathode of an electron gun is immersed in a solenoidal magnetic field, upon exit from this field, the beam has an angular momentum around the beam axis. Subsequent passage through a quadrupole channel having a 90 degree difference in phase advance between the two transverse degrees of freedom can result in a flat beam through appropriate choice of parameters.

A simple-minded version of the basic principle may be found in our paper at LINAC2000. A thorough treatment has been developed by Burov, Derbenev, and Nagaitsev.[4]

The longitudinal magnetic field profiles for the normal (round beam) and flat beam operating conditions are shown in Fig. 1. Usually, for a round beam, zero longitudinal magnetic field on the cathode is preferred in order to reduce the effective emittance arising from its angular momentum. In

\footnotetext{
* The Fermi National Accelerator Laboratory is operated under contract with the US Department of Energy
}

the present case, the longitudinal magnetic field at the cathode is needed for the transformation.

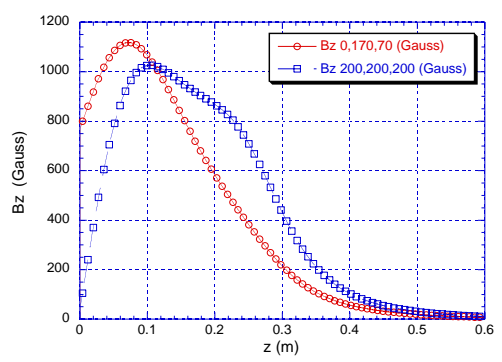

Figure 1: Longitudinal magnetic fields in the RF gun for round and flat beam operation. In the flat beam case, $\mathrm{B}_{z} \approx$ 775 gauss on the cathode.

In our procedure the transformation was accomplished with three skew quadrupoles, as illustrated in Fig 2 which depicts the layout of the Fermilab/NICADD Photoinjector Laboratory (or FNPL for short). ${ }^{1}$

\section{RESULTS}

Further analysis of the data from our running period during the Spring and Summer of 2000 has supported our conclusion reported last year that a transverse emittance ratio of about 50 has been demonstrated. This figure is limited by the diagnostics. At this point, the experiment has some distance to go to the goal of an emittance ratio of a hundred or more set by linear collider designs, as well as restoring product of the transverse emittances to that achieved under round beam conditions.

Brinkmann, Derbenev, and Flöttmann speak of an achievable emittance ratio of order $10^{2}$ or more for a beam with normalized emittance $\sqrt{\epsilon_{x} \cdot \epsilon_{y}} \approx 1 \mu \mathrm{m}$ at $1 \mathrm{nC}$ of bunch charge. The expression for the emittance ratio is

$$
\frac{\epsilon_{x}}{\epsilon_{y}} \approx \frac{4 k^{2} \sigma_{c}^{2}}{\sigma_{c}^{\prime 2}}
$$

where

$$
k \equiv \frac{1}{2} \frac{B_{z}}{\left(p_{0} / e\right)} .
$$

Here, $B_{z}$ is the field on the cathode, $p_{0}$ is the momentum at entry to the quadrupole channel, and $\sigma_{c}, \sigma_{c}^{\prime}$ are the standard deviations of the distribution in displacement and angle at

\footnotetext{
${ }^{1}$ NICADD is the acronym for the Northern Illinois Center for Accelerator and Detector Development at Northern Illinois University.
} 


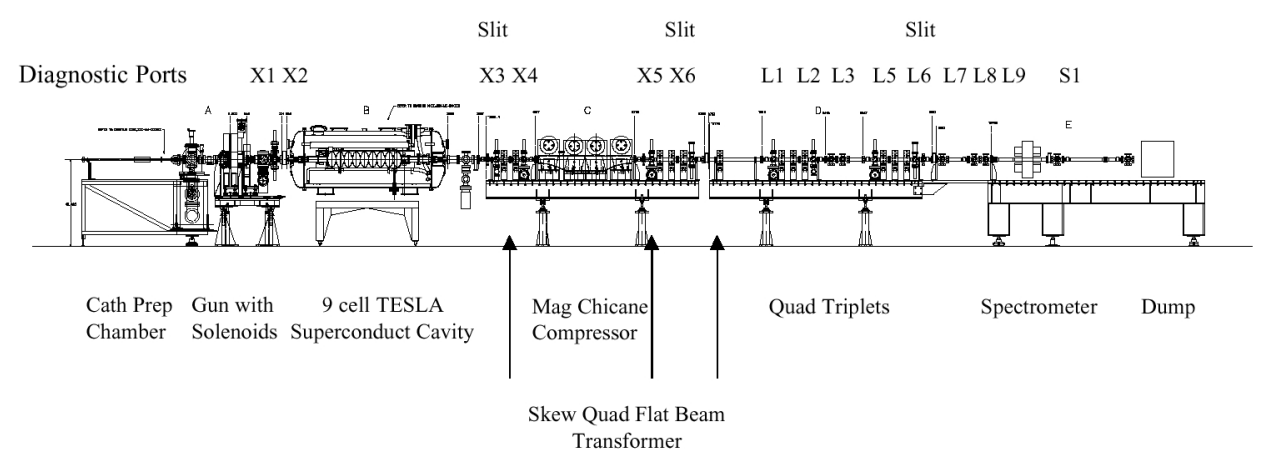

Figure 2: Layout of the FNPL photoinjector as related to this experiment.

the cathode. The resulting vertical emittance could be 0.1 $\mu \mathrm{m}$, in the range of interest for a linear collider.

The emittance measurement system consists of slits installed at three stations on the beam line. At the first of these, only a horizontal slit array is installed; the others provide slits in both horizontal and vertical orientations. Each slit array is a stack of tungsten plates of thickness $6 \mathrm{~mm}$ to the beam, with slits of width $50 \mu \mathrm{m}$ separated by $1 \mathrm{~mm}$.

The beam is observed by CCD cameras viewing aluminum-on-glass OTR screens. A sequence of images at and downstream of the first horizontal slit array with nonzero field on the cathode is shown in Fig. 3. That the beam has angular momentum is demonstrated in the apparent rotation of slit images. The associated composite beam and slit profile is shown in Fig. 4.

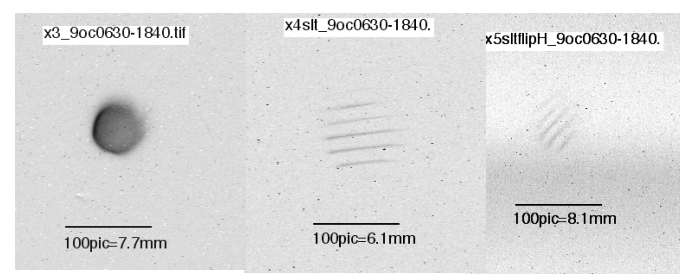

Figure 3: Beam observed at location of horizontal slit array and at successive positions downstream.

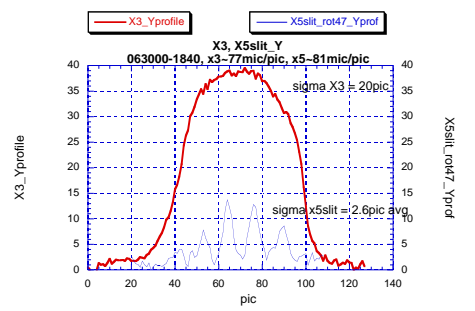

Figure 4: Beam profile at location of horizontal slit array, and slit profiles observed downstream under conditions corresponding to the preceding figure.

To produce a flat beam from the situation illustrated in Fig. 3 and Fig. 4, the skew quadrupoles are adjusted so that the long axis of the beam is oriented vertically in order to minimize depth of field problems given that the OTR screens are viewed from the side. Beam and slit images analogous to those in Fig. 3 are shown in Fig. 5;

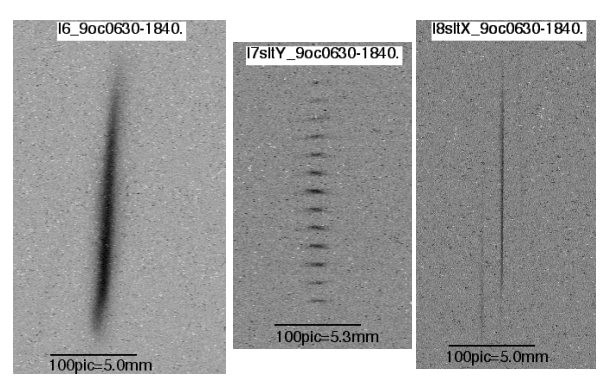

Figure 5: Beam profile at location of slit arrays, and slit images observed downstream in flat beam configuration.

In Fig. 6 we show the profiles from which vertical emittance may be obtained. In contrast, the composite profile shown in Fig. 7 reproduced from our LINAC2000 contribution illustrates the limitations of emittance measurement in the narrow dimension.

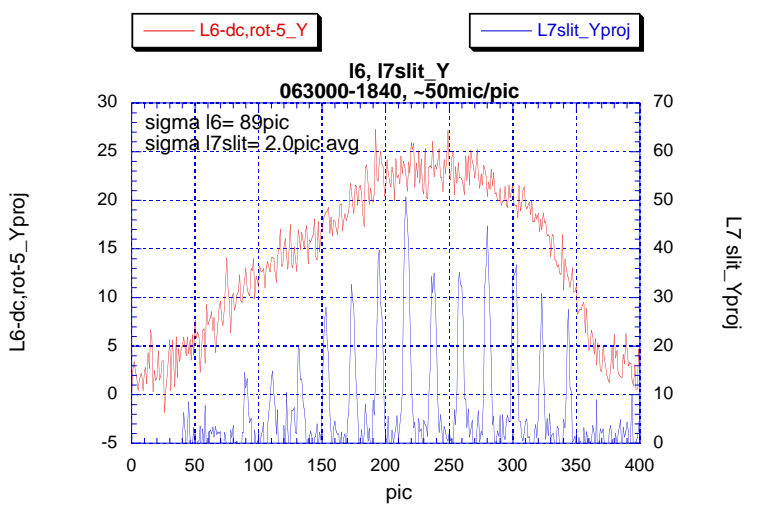

Figure 6: Slit image downstream of vertical slit array with flat beam long axis in vertical direction.

The horizontal emittance measurement is derived from a superposition of a number of pulses and is clearly resolu- 


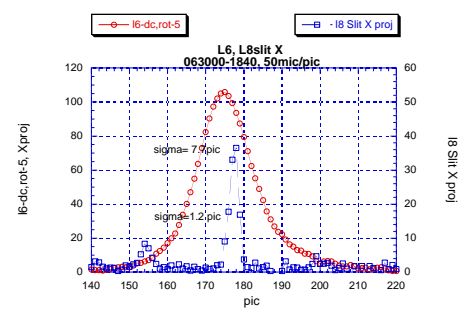

Figure 7: Projection of images used in emittance measurement at slit location and downstream of slit system.

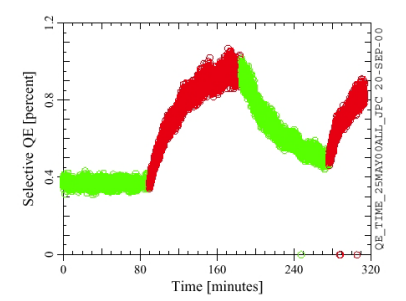

Figure 8: QE versus time; positive derivative corresponds to no longitudinal magnetic field on the cathode; negative derivative is associated with field on cathode.

tion limited. We feel that the emittance in this plane is no larger than $0.9 \mu \mathrm{m}$. The emittance in the vertical can be measured more accurately, and though it varies from day to day, the value is consistently in the mid $40 \mu \mathrm{m}$ range. Therefore, an emittance ratio of about 50 has been demonstrated, but there remains another factor of two to go in the linear collider context.

\section{PUZZLES}

- The product of the transverse emittances is higher by a factor of about 3 than is usual with operation for round beams.

- The optimum launch phase in the gun is about 20 degrees earlier than normal.

- Propagation through the superconducting booster cavity is not adequately treated in the simulations.

- Both quantum efficiency of the cathode and dark current decrease with time with our magnetic field configuration, while the reverse occurs in the normal "zero longitudinal field on the cathode state".

The last of these, the relationships among quantum efficiency, dark current, magnetic field and time, is illustrated in one aspect in Fig. 8. Further discussion of this effect may be found in the paper by Hartung et al at this conference.[6]

\section{PLANS}

Clearly we must address the items listed under the heading of Puzzles, and those all require run time and modest hardware additions.
We also wish to embark on a next phase. In their paper presented at the last year's EPAC conference in Vienna, Brinkmann, Derbenev and Flöttmann [5] predict that the combination of a long laser pulse (to reduce space charge effects at constant bunch charge) and a tailored solenoid field profile (to effect emittance compensation) will produce a significant improvement over the first experiment.

This step requires modification of the laser to stretch the pulse length while maintaining sufficient energy in the UV to produce $\mathrm{a} \approx 1 \mathrm{nC}$ bunch. Last Autumn, the pulse was extended to $30 \mathrm{ps}$ and now development of a 50 ps or longer pulse length is underway.

\section{CONCLUDING REMARKS}

Significant progress toward a high transvere emittance ratio beam has been made. But for a linear collider, a polarized electron beam is desired. Production of a beam that is both polarized and flat from an RF gun source is a nontrivial R\&D effort and these two aspects can be pursued separately to a large extent.

But even without polarization, a flat beam produced along the lines sketched here may be useful for other purposes not necessarily associated with linear colliders. Kwang-je Kim, Courtlandt Bohn and Reinhard Brinkmann have suggested benefits for the production of SmithPurcell radiation.[7] Alexander Zholents and collaborators at Lawrence Berkeley Laboratory have seen potential benefit toward their goal of production of intense femtosecond X-ray pulses.[8]

\section{ACKNOWLEGEMENTS}

Support of the Fermilab and DESY managements is gratefully acknowledged. Thanks to Jean-Paul Carneiro, Mark Champion, Michael Fitch, Joel Fuerst and Walter Hartung for their invaluable help in the operation.

\section{REFERENCES}

[1] D. Edwards et al, "The Flat Beam Experiment at the FNAL Photoinjector", Proceedings LINAC2000.

[2] R. Brinkmann, Ya. Derbenev, K. Flöttmann, "A Flat Beam Electron Source for Linear Colliders", TESLA Note 99-09, April 1999.

[3] Ya. Derbenev, "Adapting Optics for High Energy Electron Cooling”, University of Michigan, UM-HE-98-04, Feb. 1998.

[4] A. Burov, Y. Derbenev and S. Nagaitsev, "Circular Modes and Beam Adapters", submitted to Phys. Rev. E.

[5] R. Brinkmann, Y. Derbenev, K. Flöttmann, EPAC2000, Vienna, June 2000.

[6] W. Hartung et al, "Studies of Photoemission and Field Emission from an RF Photo-Innjector with a High Quantum Efficiency Photo-Cathode", Proceedings this conference.

[7] Independent private communications.

[8] A. Zholents, J. Corlett, K. Robinson, "A Dedicated Synchrotron Light Source for Ultrafast X-ray Science", Proceedings this conference. 